\begin{tabular}{|c|c|c|c|c|c|c|c|c|}
\hline \multicolumn{2}{|c|}{ Table 7.1} & \multicolumn{7}{|c|}{ Production of compound feeding stuffs } \\
\hline \multicolumn{8}{|c|}{ Monthly averages } & Thousand tonnes \\
\hline & & $\begin{array}{c}\text { Cattle and } \\
\text { calf feed }\end{array}$ & Pig feed & Poultry feed & Sheep feed & Horse feed & $\begin{array}{c}\text { Other } \\
\text { compounds }\end{array}$ & Total \\
\hline & & BFFB & BFFD & BFFE & JJ8R & JJ8S & BFFF & BFFA \\
\hline \multirow[t]{11}{*}{2007} & Feb & 333.9 & 120.7 & 206.0 & 110.4 & 17.4 & 21.5 & 810.0 \\
\hline & Mar & 384.4 & 139.3 & 248.2 & 153.6 & 19.9 & 21.5 & 966.9 \\
\hline & Apr & 305.0 & 123.2 & 217.4 & 79.6 & 14.8 & 22.5 & 762.4 \\
\hline & May & 239.2 & 126.8 & 230.7 & 32.1 & 13.4 & 26.5 & 668.7 \\
\hline & Jun & 249.1 & 145.0 & 269.9 & 25.1 & 11.9 & 33.0 & 734.0 \\
\hline & Jul & 268.8 & 133.0 & 259.2 & 21.1 & 12.5 & 34.0 & 728.6 \\
\hline & Aug & 294.2 & 134.5 & 251.8 & 25.1 & 13.0 & 36.2 & 754.7 \\
\hline & Sep & 322.6 & 147.1 & 253.8 & 25.0 & 15.7 & 36.3 & 800.6 \\
\hline & Oct & 332.1 & 137.7 & 237.1 & 24.5 & 15.9 & 30.2 & 777.6 \\
\hline & Nov & 341.3 & 132.2 & 237.4 & 31.8 & 18.5 & 25.9 & 787.2 \\
\hline & Dec & 395.2 & 144.2 & 253.8 & 51.8 & 20.6 & 25.3 & 890.9 \\
\hline \multirow[t]{12}{*}{2008} & Jan & 352.8 & 123.6 & 222.5 & 84.5 & 20.2 & 21.8 & 825.5 \\
\hline & Feb & 353.5 & 116.2 & 215.7 & 109.3 & 18.7 & 22.7 & 836.1 \\
\hline & Mar & 392.1 & 132.5 & 248.2 & 142.3 & 20.3 & 24.4 & 959.8 \\
\hline & Apr & 368.7 & 118.3 & 225.2 & 115.3 & 19.2 & 21.8 & 868.4 \\
\hline & May & 245.1 & 114.9 & 231.9 & 29.9 & 12.8 & 27.4 & 662.0 \\
\hline & Jun & 250.6 & 125.9 & 270.7 & 23.1 & 11.5 & 25.6 & 707.4 \\
\hline & $\mathrm{Jul}^{1}$ & 256.8 & 119.0 & 265.2 & 18.6 & 11.9 & 28.2 & 699.6 \\
\hline & Aug & 262.9 & 115.1 & 240.1 & 17.4 & 11.6 & 31.9 & 679.0 \\
\hline & Sep & 319.9 & 130.7 & 263.8 & 26.0 & 16.9 & 38.3 & 795.5 \\
\hline & Oct & 333.9 & 118.7 & 238.8 & 31.6 & 15.8 & 32.9 & 771.9 \\
\hline & Nov & 321.2 & 112.8 & 227.5 & 43.7 & 16.7 & 27.1 & 749.0 \\
\hline & Dec & 382.9 & 128.9 & 251.4 & 63.6 & 21.6 & 27.5 & 875.9 \\
\hline \multirow[t]{5}{*}{2009} & Jan & 327.3 & 104.7 & 210.8 & 91.3 & 17.8 & 24.9 & 776.8 \\
\hline & Feb & 315.5 & 101.8 & 198.7 & 112.8 & 17.2 & 23.8 & 769.9 \\
\hline & Mar & 369.5 & 116.9 & 242.6 & 142.1 & 19.1 & 18.7 & 908.9 \\
\hline & Apr & 295.4 & 106.4 & 221.2 & 82.6 & 15.7 & 24.5 & 745.8 \\
\hline & May & 249.4 & 105.4 & 229.8 & 36.4 & 11.8 & 27.8 & 660.5 \\
\hline
\end{tabular}

1 Revisions are possible from July 2008

Source: Department for Environment, Food and Rural Affairs: 01904455803

\begin{tabular}{|c|c|c|c|c|c|c|c|c|c|c|c|c|c|c|}
\hline \multicolumn{2}{|c|}{ Table 7.2} & \multicolumn{13}{|c|}{ Animals slaughtered and UK meat supplies } \\
\hline \multicolumn{15}{|c|}{ Monthly averages, totals for four or five week periods and quarters } \\
\hline & & \multicolumn{7}{|c|}{ Animals slaughtered (thousands) } & & & \multicolumn{4}{|c|}{$\begin{array}{l}\text { Meat - UK supplies \& Domestic Usage }{ }^{1} \\
\text { (thousand tonnes) }\end{array}$} \\
\hline & & $\begin{array}{c}\text { Steers, } \\
\text { heifers } \\
\text { and young } \\
\text { bulls }\end{array}$ & $\begin{array}{l}\text { Cows and } \\
\text { adult bulls }\end{array}$ & Calves & $\begin{array}{l}\text { Ewes and } \\
\text { rams }\end{array}$ & $\begin{array}{c}\text { Other } \\
\text { sheep } \\
\text { and lambs }\end{array}$ & $\begin{array}{c}\text { Sows } \\
\text { and adults } \\
\text { boars }{ }^{2}\end{array}$ & $\begin{array}{c}\text { Clean } \\
\text { pigs }\end{array}$ & & & $\begin{array}{c}\text { Beef } \\
\text { and veal }\end{array}$ & $\begin{array}{l}\text { Mutton } \\
\text { and lamb }\end{array}$ & Pig meat & Poultry \\
\hline & & BFHA & $\mathrm{BFHB}$ & $\mathrm{BFHC}$ & BFHD & BFHE & BFHF & BAKP & & & BFHK & $\mathrm{BFHL}$ & K8D3 & JYXE \\
\hline \multirow[t]{4}{*}{2008} & Sep $^{2}$ & 168.4 & 47.7 & 5.5 & 205.1 & $1,292.6$ & 17.9 & 724.4 & 2008 & $\mathrm{Sep}^{3}$ & 263.7 & 89.8 & 333.0 & 393.9 \\
\hline & Oct & 204.9 & 64.0 & 6.5 & 239.2 & $1,583.8$ & 21.5 & 903.9 & & Oct & .. & .. & .. & .. \\
\hline & Nov & 159.7 & 58.3 & 5.1 & 190.6 & $1,289.5$ & 16.0 & 736.8 & & Nov & .. & .. & .. & .. \\
\hline & Dec & 148.9 & 45.9 & 3.9 & 210.3 & $1,294.0$ & 13.6 & 693.4 & & $\operatorname{Dec}^{3}$ & 267.6 & 87.2 & 331.3 & 412.5 \\
\hline \multirow[t]{12}{*}{2009} & Jan & 187.1 & 50.0 & 3.4 & 206.8 & $1,259.1$ & 19.0 & 815.8 & 2009 & Jan & .. &.. & .. & .. \\
\hline & Feb & 159.1 & 38.6 & 3.0 & 170.3 & 907.8 & 16.1 & 674.1 & & Feb & .. & .. & .. & .. \\
\hline & Mar & 155.8 & 35.5 & 3.7 & 172.2 & 890.8 & 15.8 & 651.5 & & $\mathrm{Mar}^{3}$ & 246.4 & 92.6 & 332.9 & 392.7 \\
\hline & Apr & 194.8 & 40.1 & 3.2 & 200.7 & $1,057.5$ & 19.2 & 795.9 & & Apr & .. & .. & .. &.. \\
\hline & May & 155.7 & 33.7 & 2.2 & 149.8 & 762.1 & 14.6 & 624.3 & & May & .. & .. & .. & .. \\
\hline & Jun & 157.4 & 35.4 & 2.6 & 154.7 & 837.5 & 14.3 & 667.3 & & Jun $^{3}$ & 254.6 & 92.4 & 338.4 & 395.6 \\
\hline & Jul & 180.0 & 43.7 & 3.9 & 201.0 & $1,244.8$ & 19.9 & 844.5 & & Jul & .. & ... & .. & .. \\
\hline & Aug & 137.7 & 34.7 & 3.6 & 182.7 & $1,191.0$ & 16.2 & 688.8 & & Aug & .. & .. &.. & .. \\
\hline & Sep & 157.2 & 41.1 & 4.3 & 172.0 & $1,202.8$ & 17.0 & 692.5 & & $\mathrm{Sep}^{3}$ & 246.0 & 80.1 & 329.6 & 400.6 \\
\hline & Oct & 209.0 & 56.8 & 5.7 & 213.7 & $1,525.4$ & 22.0 & 908.7 & & Oct & .. &.. &.. &.. \\
\hline & Nov & 172.3 & 49.1 & 3.9 & 191.5 & $1,233.3$ & 17.3 & 739.8 & & Nov & .. & .. & .. & .. \\
\hline & Dec & 157.8 & 40.9 & 3.3 & 152.6 & $1,101.7$ & 15.2 & 721.0 & & $\operatorname{Dec}^{3}$ & 278.4 & 81.2 & 361.2 & 438.1 \\
\hline \multirow[t]{9}{*}{2010} & Jan & 186.9 & 48.3 & 2.9 & 150.0 & 959.0 & 20.1 & 844.3 & 2010 & Jan & .. & .. & .. & .. \\
\hline & Feb & 166.6 & 46.4 & 3.5 & 138.5 & 754.4 & 17.7 & 702.2 & & Feb & .. & .. & .. & .. \\
\hline & Mar & 168.8 & 40.9 & 4.3 & 131.4 & 813.9 & 18.0 & 707.3 & & $\mathrm{Mar}^{3}$ & 261.3 & 80.7 & 340.3 & 422.5 \\
\hline & Apr & 207.6 & 46.3 & 4.2 & 161.9 & 919.6 & 21.1 & 858.3 & & Apr &.. &.. &.. &.. \\
\hline & May & 167.5 & 38.6 & 2.8 & 131.3 & 691.0 & 16.3 & 694.2 & & May & .. & .. & .. & .. \\
\hline & Jun & 166.2 & 38.1 & 3.0 & 137.1 & 755.3 & 15.2 & 684.9 & & Jun $^{3}$ & 263.2 & 77.9 & 353.9 & 434.6 \\
\hline & Jul & 201.4 & 52.6 & 5.0 & 187.8 & $1,209.3$ &.. & 857.0 & & Jul &.. & .. &.. & .. \\
\hline & Aug & 158.0 & 41.0 & 5.3 & 170.5 & $1,128.2$ & .. & 717.9 & & Aug &.. &.. &.. &.. \\
\hline & Sep & 170.9 & 46.7 & 7.0 & 162.5 & $1,117.9$ & .. & 728.5 & & Sep & .. & .. &.. & .. \\
\hline
\end{tabular}

1 From January 2007, 'Meat - UK supplies \& Domestic Usage' are shown as quarters and the data are provisional.

2 July, August and September 2010 data for Sows and adult boars is confidential.

3 Data are provisional.

Source: Department for Environment, Food and Rural Affairs: 01904455803 


\begin{tabular}{|c|c|c|c|c|c|c|}
\hline Meats & roduce & erly averages & & & & Thousand tonne \\
\hline & & $\begin{array}{c}\text { Pig Meat }{ }^{2} \\
\text { Home production }\end{array}$ & $\begin{array}{l}\text { Beef and veal } \\
\text { Home production }\end{array}$ & $\begin{array}{l}\text { Mutton and lamb }{ }^{2} \\
\text { Home production }\end{array}$ & $\begin{array}{c}\text { Poultry Meat } \\
\text { Home cured production }\end{array}$ & $\begin{array}{c}\text { All Meat } \\
\text { Home production }\end{array}$ \\
\hline & & K8BY & IFY9 & IFZ2 & IFZ3 & JJ8XX \\
\hline 2002 & & 774.4 & 694.3 & 307.3 & 1557.0 & 3333.1 \\
\hline 2003 & & 687.9 & 703.2 & 309.8 & 1557.1 & 3258.0 \\
\hline $2004^{4}$ & & 678.6 & 723.6 & 319.2 & 1543.0 & 3264.6 \\
\hline 2005 & & 669.3 & 764.8 & 336.7 & 1570.2 & 3340.9 \\
\hline 2006 & & 667.5 & 852.1 & 333.5 & 1511.9 & 3365.0 \\
\hline $2007^{3}$ & & 706.7 & 888.5 & 329.5 & 1466.4 & 3391.0 \\
\hline 2008 & & 705.7 & 866.0 & 331.6 & 1464.4 & 3367.6 \\
\hline $2009^{3}$ & & 681.1 & 840.7 & 309.1 & 1459.3 & 3290.2 \\
\hline 2005 & Q2 & 163.1 & 191.3 & 70.6 & 381.2 & 806.3 \\
\hline & Q3 & 164.9 & 190.9 & 89.1 & 389.7 & 834.6 \\
\hline & Q4 & 173.5 & 204.3 & 97.1 & 397.2 & 872.0 \\
\hline 2006 & Q1 & 164.6 & 206.8 & 83.5 & 372.9 & 827.8 \\
\hline & Q2 & 158.1 & 207.1 & 69.5 & 381.9 & 816.6 \\
\hline & Q3 & 167.7 & 209.4 & 87.9 & 369.9 & 834.9 \\
\hline & Q4 & 177.1 & 228.8 & 92.6 & 387.3 & 885.7 \\
\hline 2007 & Q1 & 173.8 & 228.0 & 75.9 & 363.3 & 840.9 \\
\hline & Q2 & 170.7 & 220.3 & 76.4 & 363.9 & 831.3 \\
\hline & Q3 & 169.7 & 209.6 & 81.9 & 360.8 & 822.1 \\
\hline & Q4 & 192.4 & 230.6 & 95.3 & 378.4 & 896.7 \\
\hline 2008 & Q1 & 180.9 & 221.7 & 83.8 & 359.1 & 845.5 \\
\hline & Q2 & 171.3 & 214.3 & 68.3 & 359.2 & 813.0 \\
\hline & Q3 & 174.7 & 207.5 & 86.8 & 368.1 & 837.1 \\
\hline & Q4 & 178.8 & 222.5 & 92.7 & 378.0 & 872.0 \\
\hline 2009 & $\mathrm{Q} 1^{3}$ & 165.2 & 207.5 & 72.2 & 353.7 & 798.7 \\
\hline & $\mathrm{Q} 2^{3}$ & 159.7 & 206.4 & 64.7 & 356.6 & 787.4 \\
\hline & $\mathrm{Q}^{3}$ & 171.2 & 197.1 & 83.9 & 360.9 & 813.1 \\
\hline & $\mathrm{Q} 4^{3}$ & 185.0 & 229.6 & 88.3 & 388.0 & 891.0 \\
\hline 2010 & $\mathrm{Q} 1^{3}$ & 176.3 & 223.2 & 60.7 & 374.7 & 834.9 \\
\hline & $\mathrm{Q} 2^{3}$ & 171.8 & 227.3 & 57.7 & 376.8 & 833.6 \\
\hline
\end{tabular}

1 Does not include meat offals or trade in preserved or manufactured meat products (eg: canned meat).

2 Includes an estimate of the dressed carcase weight of live exports other than pure bred.

3 Data are provisional.
4004 home-fed production figures were collected on a 53 week ( 14 week 4th Quarter) basis but have been adjusted to a 52 week year (13 week 4th quarter) for comparability.

Source: Department for Environment, Food and Rural Affairs: 01904455803

\section{Table 7.4}

Milk and milk products

Monthly averages or calendar months

\begin{tabular}{|c|c|c|c|c|c|c|c|c|c|}
\hline & \multicolumn{4}{|c|}{ Million litres } & \multicolumn{4}{|c|}{ Thousand tonnes } \\
\hline & & \multicolumn{4}{|c|}{ Disposals of raw milk for manufacture } & \multicolumn{4}{|c|}{ Wholesale production ${ }^{2}$} \\
\hline & & Liquid milk ${ }^{1}$ & Total & Exports & $\begin{array}{l}\text { Stock change } \\
\& \text { wastage }\end{array}$ & $\begin{array}{l}\text { Condensed } \\
\text { milk }^{3}\end{array}$ & Milk powders ${ }^{3,4}$ & Butter & Cheese \\
\hline & & IFX4 & IFX5 & IFX6 & IFX7 & IFX8 & IFX9 & IFY2 & IFY3 \\
\hline 2004 & & $6,693.3$ & $6,723.9$ & 434.3 & 106.9 & 161.2 & 167.6 & 121.7 & 351.3 \\
\hline 2005 & & $6,652.5$ & $6,489.8$ & 626.3 & 86.1 & 142.7 & 121.5 & 130.0 & 384.2 \\
\hline 2006 & & $6,734.3$ & $6,266.3$ & 617.3 & 99.7 & 113.3 & 114.1 & 117.1 & 393.3 \\
\hline 2007 & & $6,723.7$ & $6,085.1$ & 537.6 & 126.8 & 109.2 & 117.0 & 120.4 & 372.7 \\
\hline 2008 & & $6,678.3$ & $5,840.0$ & 559.2 & 91.5 & 109.9 & 94.3 & 111.1 & 382.0 \\
\hline 2009 & & $6,626.4$ & $5,699.4$ & 433.4 & 293.4 & 104.3 & 101.7 & 117.6 & 357.1 \\
\hline \multirow[t]{4}{*}{2008} & Sep & 542.0 & 413.8 & 42.8 & -8.3 & 26.0 & 19.8 & 9.2 & 28.4 \\
\hline & Oct & 570.7 & 408.5 & 55.6 & -0.8 & .. & .. & 9.7 & 29.4 \\
\hline & Nov & 565.7 & 384.3 & 53.0 & 4.0 & 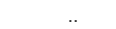 & .. & 8.9 & 26.2 \\
\hline & Dec & 579.9 & 456.7 & 38.1 & -5.8 & 28.3 & 14.4 & 8.4 & 28.5 \\
\hline \multirow[t]{12}{*}{2009} & Jan & 560.6 & 460.3 & 38.5 & 30.8 & .. & .. & 11.0 & 29.4 \\
\hline & Feb & 513.8 & 430.0 & 34.4 & 21.2 & .. & .. & 10.9 & 25.8 \\
\hline & Mar & 571.1 & 500.9 & 41.2 & 29.9 & 26.0 & 27.0 & 11.5 & 31.2 \\
\hline & Apr & 548.9 & 542.6 & 41.7 & 26.0 & .. & .. & 11.2 & 33.7 \\
\hline & May & 569.2 & 578.5 & 41.2 & 32.0 & .. &.. & 11.6 & 35.4 \\
\hline & Jun & 538.4 & 547.0 & 36.4 & 22.9 & 25.5 & 35.0 & 10.3 & 34.8 \\
\hline & Jul & 567.1 & 489.9 & 41.9 & 18.5 & .. & .. & 9.4 & 31.9 \\
\hline & Aug & 554.4 & 462.3 & 33.4 & 19.2 &.. &.. & 8.6 & 30.6 \\
\hline & Sep & 558.1 & 412.6 & 29.2 & 8.4 & 25.1 & 19.5 & 7.6 & 27.2 \\
\hline & Oct & 553.7 & 427.9 & 29.2 & 27.3 & .. & .. & 8.1 & 27.5 \\
\hline & Nov & 536.1 & 399.6 & 32.6 & 30.0 & .. &.. & 8.8 & 23.5 \\
\hline & Dec & 555.0 & 448.0 & 33.7 & 27.2 & 27.8 & 20.2 & 8.6 & 26.1 \\
\hline \multirow[t]{8}{*}{2010} & $\operatorname{Jan}^{5}$ & 584.6 & 440.4 & 36.2 & 3.5 &.. &.. & 10.4 & 27.2 \\
\hline & $\mathrm{Feb}^{5}$ & 529.2 & 426.1 & 33.7 & 2.2 &.. &.. & 9.8 & 27.7 \\
\hline & $\mathrm{Mar}^{5}$ & 589.8 & 499.0 & 36.5 & 7.1 & 25.2 & 18.7 & 10.1 & 32.3 \\
\hline & $\mathrm{Apr}^{5}$ & 554.2 & 544.5 & 38.0 & 11.9 &.. &.. & 11.1 & 32.8 \\
\hline & $\mathrm{May}^{5}$ & 599.0 & 614.8 & 37.5 & 9.0 & 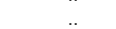 &.. & 11.3 & 36.6 \\
\hline & $\operatorname{Jun}^{5}$ & 557.9 & 596.4 & 33.0 & 7.1 & 24.6 & 36.8 & 11.3 & 37.1 \\
\hline & $\mathrm{Jul}^{5}$ & 583.6 & 527.2 & 36.5 & 8.0 & & .. & 9.7 & 32.8 \\
\hline & Aug $^{5}$ & 571.9 & 511.6 & 34.5 & 3.5 & & & 9.5 & 32.6 \\
\hline
\end{tabular}

1 Liquid milk that leaves the primary dairy in liquid form. This may be used for manufacture of other food products, or as liquid drinking milk. 2 Excludes production by direct sellers of liquid milk, butter and cheese (ie. Farmhouse production).

3 Monthly figures are not available from 2006 onwards due to confidentiality issues.

4 Full cream, whole milk, partially skimmed milk and skimmed milk powders.

5 Provisional data, may be subject to revision.

Source: Department for Environment Food and Rural Affairs: 01904455803 


\section{Table 7.5}

Cereals and cereal products

Monthly averages or totals for four or five week periods. Stocks refer to the end of the period

Thousand tonnes

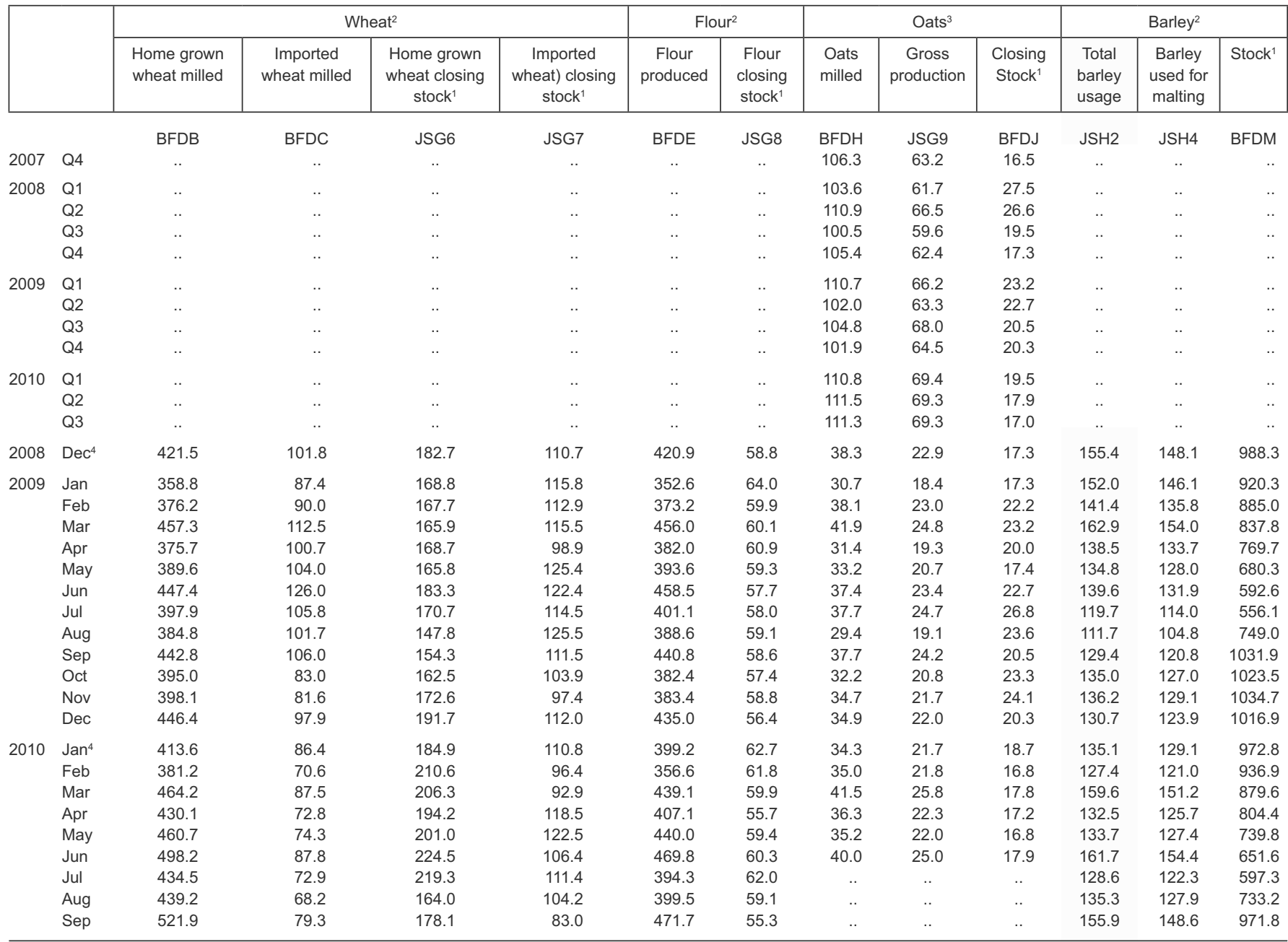

Note: Due to rounding totals may not agree with the sum of the individual items.

1 Stocks held by wheat millers, feed compounders, cereal breakfast food manufacturers, brewers, maltsters and distillers, merchants and dealers.

2 Figures for Wheat, Flour and Barley are only available monthly.

3 Monthly figures for Oats are not available after June 2010.

4 There were 53 weeks in the Statistical year 2010. In order to incorporate the change January 2010 was increased to a 5 week period compared to 4 weeks in 2009 .

Source: Department for Environment, Food and Rural Affairs: 01904455803 
Tobacco products released for home consumption

Monthly averages or calendar months

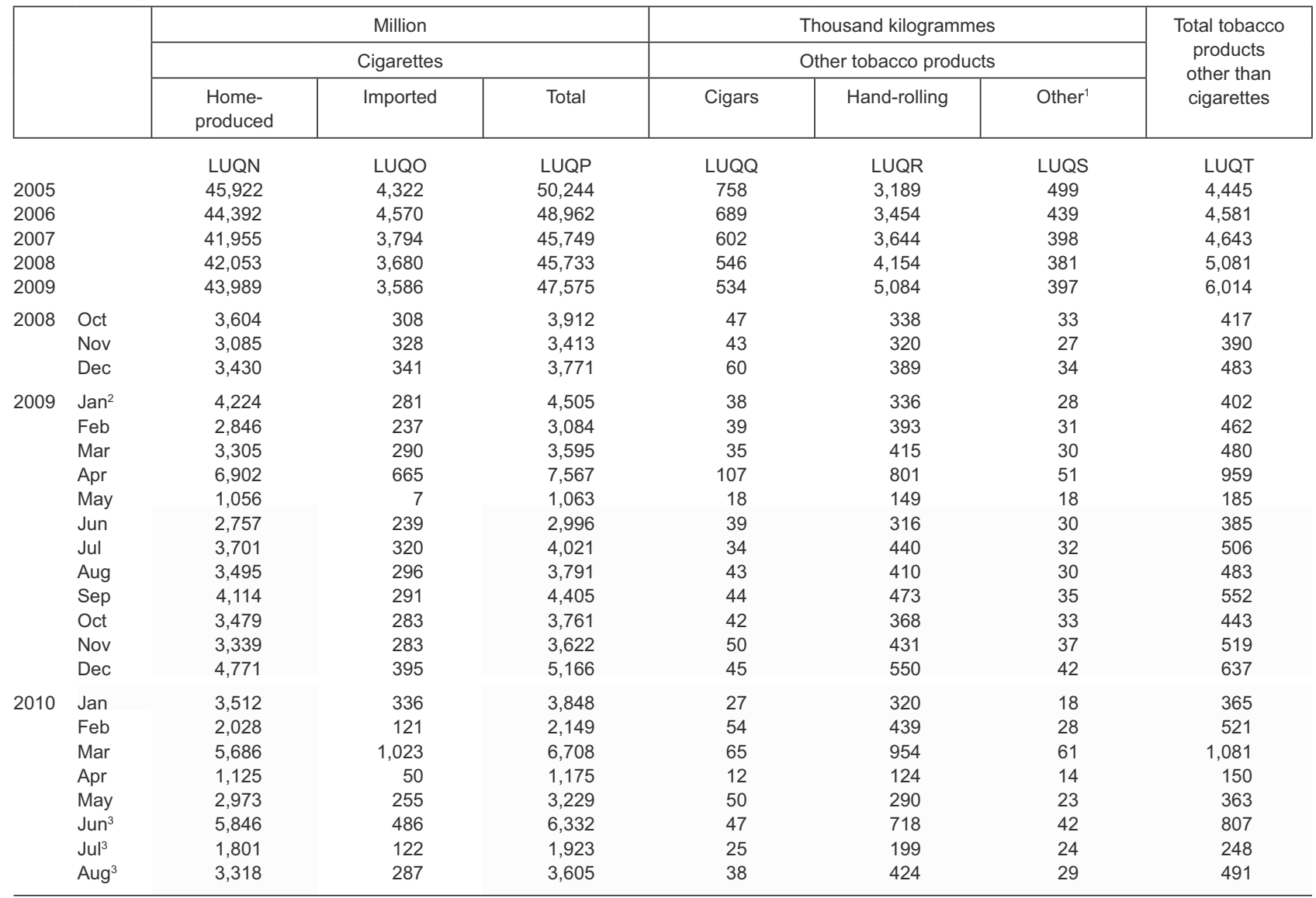

Note: Releases of cigarettes and other tobacco products tend to be higher in the period before a Budget. Products may then be stocks, duty paid, before being sold.

1 Excluding snuff.

2 Due to the effect of the holiday period, these figures are subject to greater uncertainty than usual.

3 Provisional

4 Revisions are possible from July 2010

Sources: HM Revenueand Customs Statistical Bulletins at;

http://www.uktradeinfo.com/index.cfm?task=bulletins 


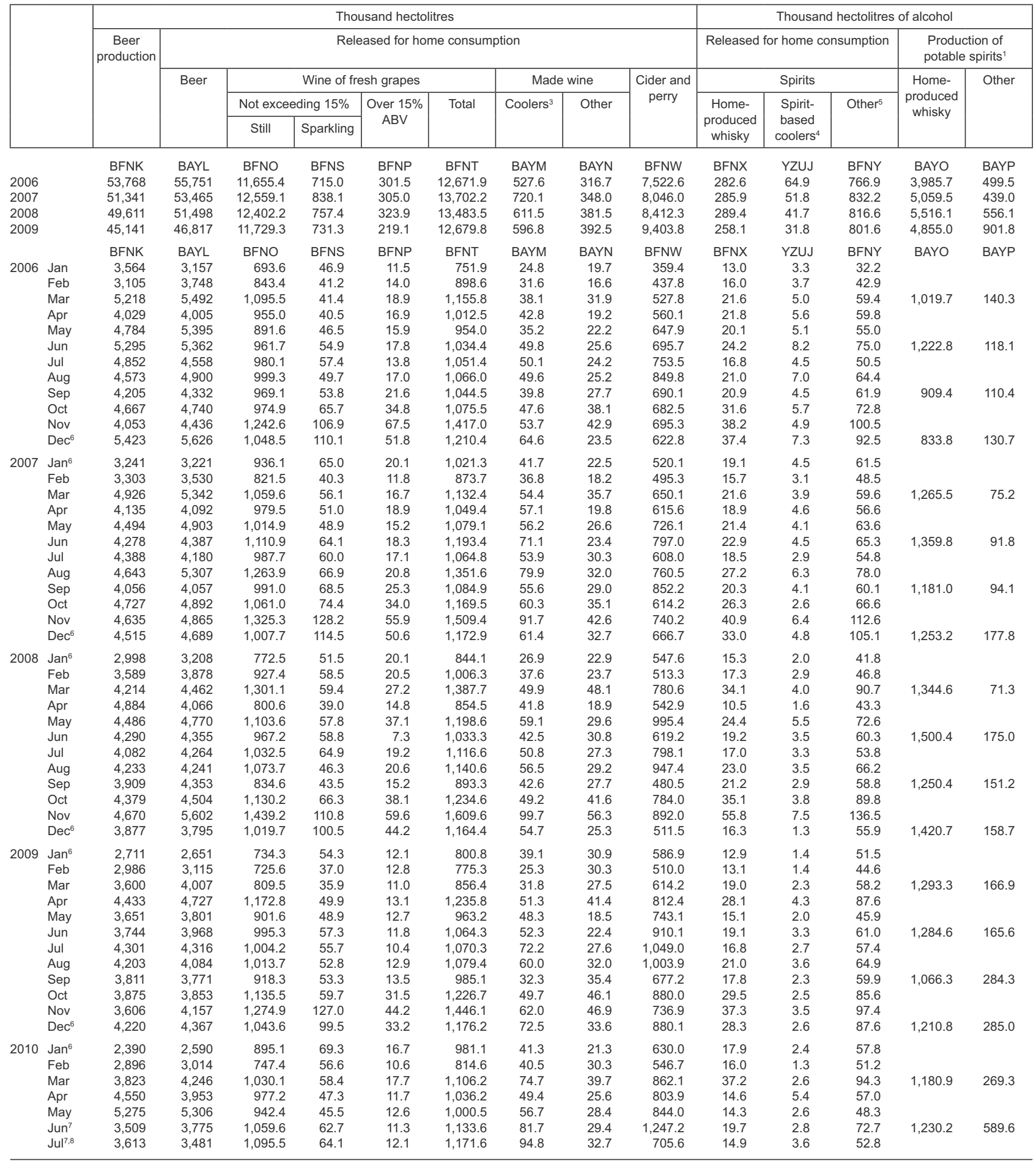

Note: some figures have seen small revisions since they were last published.

1 Data are available only quarterly and in the months following the quarter.

Percentage alcohol by volume.

3 Made wine with alcoholic strength $1.2 \%$ to $5.5 \%$, includes alcoholic lemonade of appropriate strength.

4 From 28 April 2002 duty on spirit-based ready-to-drink (RTDs) products is charged at the same rate as spirits per litre of alcohol. Spirit-based RTDs were previously dutied at the made wine rate and details on quantities can be found in made wine coolers.

5 Includes imported spirits.

6 Due to the effect of the holiday period, these figures are subject to greater uncertainty than usual. Also, unusually high or low figures may be changed on receipt of amendments to returns data.

7 Provisional

Revisions are possible from July 2010

Source: HM Revenue and Customs Alcohols Bulletins at: https://www.uktradeinfo.com/index.cfm?task=bullAlcohol 
Index numbers of textile and clothing industries

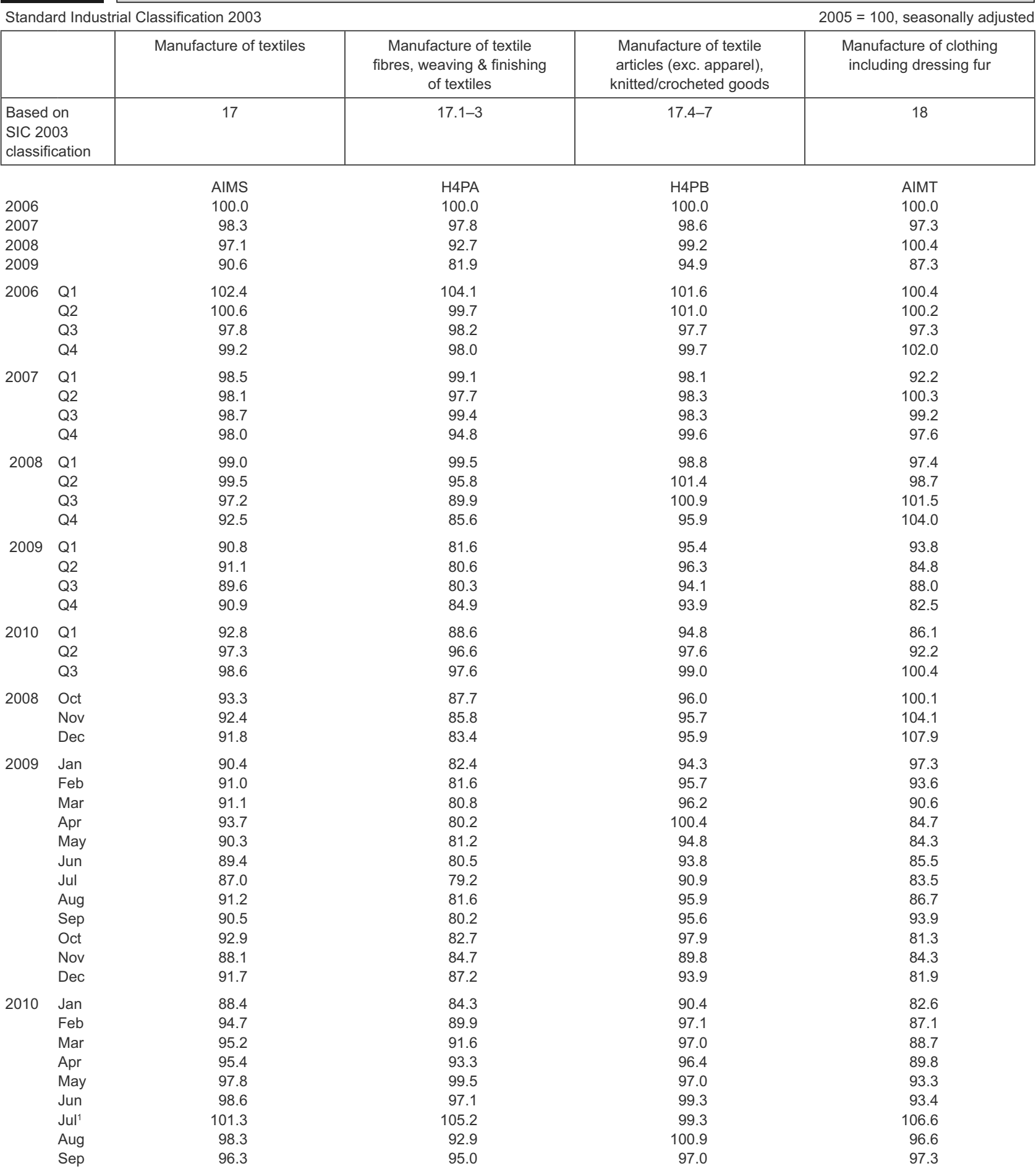

Note: ONS has introduced metholological changes to the Index of Production from March 2008 (January 2008 data). Also, a new industry structure means that the level of detail published is reduced. For the textile and clothing sector, industries are no longer available at the full level of disaggregation. Further information can be found on the NS website at - http://www.statistics.gov.uk/cci/article.asp?id=1908

1 Revisions are possible from July 2010

Source: Office for National Statistics: 01633455803 